\title{
Occupational Therapy with the Military Upper Extremity Amputee: Advances and Research Implications
}

\author{
Josef Butkus • Caitlin Dennison • Annemarie Orr • \\ Matthew St. Laurent
}

Published online: 9 November 2014

(C) Springer Science+Business Media New York (Outside the USA) 2014

\begin{abstract}
Conflicts in Iraq and Afghanistan have resulted in an increased number of United States service members (SM) with upper extremity amputations, resulting in new prostheses and increased research in the field. As of July 2014, there have been 1,648 patients suffering limb loss since the start of the conflicts, 511 of which involve multiple limb amputations (Military Amputee Patient Care Program Database, Extremity Trauma and Amputation Center of Excellence, 2014). Walter Reed National Military Medical Center has seen 1,224 of 1,648 returning SM with amputations. Of the total number of injuries, 287 traumatic amputations or $17.4 \%$ of these involve an upper extremity (Military Amputee Patient Care Program Database, Extremity Trauma and Amputation Center of Excellence, 2014). Increased military support and funding have led to the advancement of research and development of new technologies. Occupational therapy amputee care has evolved and been documented in publications outlying treatment protocols that describe rehabilitation with this population (Smurr et al., J Hand Ther, 21(2):160-176, 2008). This article will serve as an overview of the current state of rehabilitative care for the military upper extremity amputee, implications for care, advances in the field, and research needs and initiatives.
\end{abstract}

Keywords Occupational therapy $\cdot$ Military $\cdot$ Upper extremity amputee

J. Butkus $(\bowtie) \cdot$ C. Dennison · A. Orr · M. St. Laurent Occupational Therapy, Amputee Section Supervisor, Walter Reed National Military Medical Center, Bethesda, MD 20889, USA

e-mail: Josef.Butkus@med.navy.mil

\section{Acute Care}

Conflicts in Iraq and Afghanistan have resulted in 1,648 patients suffering limb loss, 511 of which involve multiple limb amputations [1]. Walter Reed National Military Medical Center has seen 1,224 of these 1,648. Of these injuries, 287 amputations or $17.4 \%$ involve an upper extremity [1]. Occupational therapy amputee care continues to evolve, leading to the development of a treatment protocol that describes rehabilitation with this population [2••]. The first phase of rehabilitation following limb loss begins with Acute Care. The acute phase of occupational therapy (OT) amputee care begins with a comprehensive evaluation of the patient. Physical therapy (PT) works hand in hand throughout the course of rehabilitation. A baseline physical and functional assessment post injury is an important indicator of the patient's progress and outcomes during recovery. This includes patient history (past medical history, home and social environment, premorbid functional level), physical assessment of the involved and contralateral extremity (range of motion, strength, sensation, balance), and functional assessment including activities of daily living (ADLs), mobility, and cognition.

Pain management is immediately addressed during the post-operative stages of healing and continues throughout rehabilitation. Early pain management prevents the development of chronic pain for upper extremity amputations. When pain is not controlled it interferes with therapy participation, sleep, length of inpatient stay and leads to poor compliance with prosthetic fitting/training. Residual limb pain and phantom limb pain are the two common types of pain. Residual limb pain occurs in the remaining part of the amputated limb while phantom pain is perceived as occurring in the missing part of the limb. Pain control is mostly addressed through administration of pharmaceuticals, but 
therapists also play a significant role. OT treatment modalities to address post-amputation pain include desensitization and massage of the residual limb, edema management, heat/ ice contrast baths, transcutaneous electrical nerve stimulation (TENS), visual imagery, and mirror therapy [3]. OT plays a significant role in limb desensitization prior to an initial prosthesis fitting, as well as facilitating patient acceptance of the altered limb.

Prior to being fit for an initial prosthesis, the residual limb needs wound closure. Patient education for residual limb care is critical in this phase. Education is provided on proper donning/doffing techniques of compression devices, daily skin inspection for breakdown or abnormal pressure, and proper limb hygiene. Shrinkers or ace wraps are used early on to reduce edema and shape limbs for optimal prosthetic fitting. Fitting a patient with prosthesis within 4 weeks after amputation will increase chances of acceptance of the prosthetic limb [4]. This is known as the 'golden period' of upper extremity prosthetic rehabilitation and may be the most vital factor in the patients' acceptance of the prosthesis [4].

Contractures and muscle atrophy are common risk factors that can affect prosthetic use and overall function. Range of motion and strengthening the BUE musculature is essential in maintaining body symmetry, posture, and alignment of the upper quadrant to reduce overuse injuries. Within weeks of an amputation, therapists and prosthetists must identify the residual limb myosites to initiate myoelectric training. A biofeedback unit, similar to those used in myoelectic prostheses is used to identify EMG signals. The available muscles are trained to promote prosthetic control for immediate reliable function once they receive the prosthesis.

ADL training is initiated as early as possible in the acute phase of rehabilitation, to facilitate a return to independence in basic daily tasks. Performing self-feeding, hygiene and toileting with universal cuffs, splints, and devices secured to the residual limb allows the patient to regain some control over their environment. Additionally, assistive technology is used to make a patient hospital room more accessible. Examples include call bells to contact nursing staff, patient controlled analgesic (PCA's), and adaptive devices for phones and computer use. In this acute phase, OT encourages the patient to attempt to be independent with as many activities as can be tolerated, incorporating the amputated upper extremity if possible.

\section{Advanced Outpatient}

When the patient is ready to transition to outpatient OT, preprosthetic training is continued until the patient is ready for the initial prosthesis. The initial prosthesis is either body powered or myoelectric powered. Typically, a myoelectric prosthesis is recommended for initial training, in order for the patient to start strengthening the muscles needed to control it, as the training is more complex. A myoelectric prosthesis uses small sensors inside the socket that contact the skin over the remaining musculature. Contracting the muscle powers the terminal device to open or close. However, the recommendation of the initial prosthesis is dependent on the patient's residual limb appropriateness, needs and close collaboration with the prosthetist. Co-morbidities such as nerve damage or other injuries, in which the patient is unable to perform muscle contractions needed for a myoelectric, may result in a body-powered prosthesis being the initial recommendation. A body-powered prosthesis has a cable that runs from the straps on the body down to the terminal device, in which scapular protraction and shoulder flexion result in the opening or closing of the terminal device. Prosthetic training begins with basic control and progresses to refined control. The initial goal of therapy is basic control, in which the patient practices open/close of the terminal device, and other components that may be included for this prosthesis (Wrist and/or elbow).

When the patient masters these movements, the next step is object manipulation and incorporation into tasks. Initial objects are large and evenly shaped for gross grasp (cups and blocks) gradually progressing to smaller and more irregularly shaped items for advanced fine motor training (pegs and pins). Rote training is emphasized as this creates natural movement by improving dexterity, coordination, and control. Pre-positioning the terminal device is not always intuitive for the patient, and takes practice to learn. Another goal of object manipulation is grip control, in which the patient learns how much pressure to exert with the terminal device, based on the density and durability of the object (soft vs firm items). As the patient has no sensation through the terminal device, the patient must rely heavily on visual feedback to determine if an item is being held with appropriate grip force. With practice in therapy, eventually the patient can operate the prosthesis successfully without consistent visual regard. Proportional control is related to controlling variable speed, which is related to efficient task performance. These methods of object manipulation are introduced to whole tasks and analysis of prosthetic inclusion begins.

Once basic control of the prosthesis is mastered, functional tasks with increasing complexity are incorporated into treatment. Although the above is the general training guide, therapy is structured according to patient ability and goals. When treating a patient with bilateral upper extremity amputations, the first therapy tasks are basic ADLs such as toileting or feeding, as the primary goal is immediate autonomy with self-care. Patients with unilateral upper extremity amputations quickly master one-handed techniques for ADL performance and tend to incorporate their prosthesis for bilateral tasks or as an assist to the intact limb. As the therapy focus of prosthetic 
training transitions to functional tasks, the therapist educates the patient about appropriate inclusion of the prosthesis into each task. Anticipatory reactions that one would perform with an intact limb, such as positioning the limb and which task the prosthetic limb is performing, can eventually become automatic with a prosthesis for a skilled user. Household tasks, community reintegration, and return to work become increasingly important during the final stages of prosthetic training. A comprehensive ADL task list is utilized in therapy sessions with pre-identified ADLs and instrumental activities of daily living (IADLs). IADLs are higher level complex skills such as cooking, shopping, and laundry that are needed to live independently. An excellent guideline to this is the 'Unilateral Upper Extremity Amputation: Activities of Daily Living Assessment,' a task list that the patient performs throughout therapy sessions while the therapist qualitatively rates how smoothly these tasks are performed on a scale of 0-3 [5・•]. Additionally, the therapist and patient concurrently set goals based on the patient roles (spouse, parent, employee) and incorporate the resulting tasks into therapy sessions. This allows the patient to trial various types of prostheses and terminal devices for different tasks and determines appropriateness and method of incorporation.

Over time, patients become adept at task analysis and identifying barriers to accomplishing certain activities. Being able to independently determine which prosthesis and terminal device is most useful for a particular task is an important therapeutic goal. At this point, patients are introduced to advanced terminal devices that have more complexity and control. Advanced prosthetic training at Walter Reed National Military Medical Center (WRNMMC) typically involves myoelectric training with the newer multiarticulating hands such as the Bebionic and the I-limb. The prosthetist, therapist, and patient discuss goals and recommend appropriate advanced terminal devices. Patients assist in driving this process to determine their needs and preferences through trial, acquisition, and training of advanced terminal devices.

Once the patient has trialed multiple prosthetic options, selecting a device for a particular activity becomes second nature. At this point, the patient determines what terminal devices and prosthetic systems are needed for daily life and what systems are needed for specific activities. Activity-specific terminal devices are then selected based on the patient's needs and interests related to sports, hobbies, and other activities. Collaboration with recreation therapists is important to ensure successful use and training of these devices in adaptive sports and community reintegration outings.

Although patients are educated about various prosthetic options, each patient makes individualized choices. Some patients choose to only use myoelectric prostheses or bodypowered prostheses, while others choose not to use prostheses at all. For unilateral upper extremity amputees, patients are more able to perform activities without prostheses as there is an intact limb. For bilateral upper extremity amputees, using at least one prosthesis is necessary in order to achieve more independent function. As many of the bilateral upper extremity amputees at WRNMMC were also missing at least one lower limb, upper extremity prostheses are necessary in order to independently don/doff the lower prostheses and to assist with ambulation by grasping/incorporating an ambulatory assistive device, such as a cane. General patterns of prosthetic choices were noted based on level of amputation. Patients with transradial amputations tended to be more consistent prosthetic users, typically myoelectric. Patients with amputations above the elbow were more likely to reject prosthetic use per patient report due to discomfort and weight. Myoelectric prostheses for above elbow amputees increase difficulty of control due to inconsistent myo signals which can be due to increased soft tissue or movement of the limb within the socket. If these patients do choose to be prosthesis users, a body-powered prosthesis is the typical choice as it is more light weight and has more reliable control per patient reports. Among those who are prosthetic users, some are 'cosmetic' users (grossly using a passive hand to stabilize objects for specific tasks without a functional pinch) while other patients are mechanical users, (involving the terminal device in all parts of a particular task). Among those who are mechanical users, prosthetic preferences also vary widely for socket, suspension, internal lining, and terminal devices.

Although OT performs prosthetic training, the overarching goal of OT with this population is independent performance of all ADLs and IADLs, both with and without prosthesis. Prostheses are tools to increase function and the OT provides the patient with education on each of those tools, so that when presented with any activity the patient will be able to participate successfully and to their satisfaction. The most crucial aspect of this care is ensuring the quest for independence is patient-driven and incorporates the patient's individual goals and preferences. Quality of life is of upmost importance no matter which type of prosthesis they may use or choose not to wear, but OT can serve to increase success with the prosthesis and acceptance.

\section{Assessment}

As there are multiple factors considered when determining success with prosthetic use, the OT assessment of upper extremity prosthetic use presents unique challenges. These factors of success include quality of life, appropriate incorporation, cosmesis, dexterity, accuracy of control, comfort and tolerance, internal feedback, body positioning, 
and frequency of use. Success in balancing these factors is most effectively evaluated through qualitative OT assessment. For the amputee population, this is the best way to constructively analyze performance.

Several OT evaluation tools are commonly used to assess functional performance with a prosthesis. Virginia Wright organized and summarized all of these assessments in her 2009 systematic review of prosthetic outcome measures article [6]. The Assessment of Capacity for Myoelectric Control (ACMC) assesses nuances of performance with a myoelectric prosthesis while gripping, holding, releasing, and readjusting the prosthesis, both with or without explicit vision [7]. The Jebsen-Taylor Test of Hand Function is not standardized for a prosthesis, however, remains useful to assess task performance and dexterity [8]. The Box and Blocks Test [9] and the Nine Hole Peg Test [10] are dexterity assessments, also not standardized for a prosthesis. The Southampton Hand Assessment Procedure (SHAP) examines dexterity with performance of a number of tasks requiring different pinches and grips and norms them in an Index of Functionality Scale [11]. The comprehensive ADL/ IADL task list [5••] previously mentioned has been tremendously useful in determining dexterity and quality use through a more qualitative means. Despite the lack of a single comprehensive assessment, the currently available assessments allows therapists to identify areas of success and opportunities for the patient's performance to improve.

Although there is no comprehensive test that can objectively define success across multiple factors of performance, it is still possible to examine the benefit for prosthetic use. Prosthetic use decreases the burden on the contralateral limb, increases function by offering an additional grasp with bimanual tasks, assists in body symmetry, and improves self-image. The ongoing qualitative analysis of performance guides therapy. OT's assess aspects of performance that can be improved, foster independent problem solving tasks, provide guidance to determine preferences, improve dexterity and control, and assist patients in appropriate incorporation of prostheses in specific tasks. These interactions between therapist and patient are vital to promote successful performance with a prosthesis. The development of standardized assessment for upper extremity prosthetic use would serve to identify standards of performance, ensure quality treatment, guide therapy, improve patient performance, and potentially affect quality of life.

\section{Advanced Terminal Devices Overview}

As technology evolves, the variety of advanced terminal devices available to prosthetic users has expanded. Myoelectric terminal devices are the main focus of this area as these are the most technologically advanced. Currently the most widely used new devices are the iLimb and the Bebionic. Traditional myoelectric terminal devices only offer the option for open/close of the hand. Both devices articulating digits offer over ten grasp patterns to choose from, including custom hand positions for the iLimb. The thumb can be placed in lateral positions to allow a larger variety of grasp patterns. The iLimb ultra revolution offers powered opposition of the thumb. The Sensor Hand Speed from Otto Bock also offers an anti-slip feature, such that if an item in the hand begins to slip, sensors power the digits to tighten the grip and prevent dropping the items. There are a variety of wrist control options available for these terminal devices to allow for active pronation/supination. Appearance is improved by the availability of semi-custom and custom silicone covers. Users generally prefer the option for various pinch and grasp patterns, and also generally find these terminal devices to be more esthetically pleasing. However, with advanced technology comes decreased durability, when compared to less complex terminal devices or body-powered options. The iLimb or Bebionic terminal devices appears to be most appropriate for unilateral upper extremity amputees versus bilateral upper extremity amputees, as unilateral users are able to use the device as an assist to their intact side. These advanced prostheses are made to be more mobile and not as reliable with heavy duty tasks such as transfer assists and gait training with assistive devices. Additionally, the fine motor pinch force on these devices is significantly less than traditional devices, which makes some tasks difficult as objects are more likely to slip out of the fingers. These terminal devices are the most advanced that are commercially available, although other devices are currently being researched/ trialed. For example, the Vincent hand [12] is another advanced myoelectric terminal device that is available, however, has not yet been trialed or used at this facility. Advanced myoelectric prostheses offer improved cosmetics and grasp patterns, but may not be the best choice for all patients.

There are also a variety of myoelectric advanced digits for partial hand amputees, such as the iLimb digits. These are operated in a similar fashion with individually powered articulating digits. Fitting varies widely as it is dependent on the remaining anatomy; hence the functional use of myoelectric digits is extremely individualized. The hardware and battery must be housed in the socket which can result in a bulky prosthesis. The Vincent fingers [13] are another myoelectric digit option which has not yet been trialed at this facility. There are also body-powered options for individual fingers systems exist as well, such as products like the M-fingers [14]. Some barriers for using these systems are durability and socket position, which often compromises sensation at the distal end of the residual limb and impacts use. In general, a large number of patients at WRNMMC with partial hand amputations have rejected 
the use of prostheses. However, patients who have been most successful in incorporating digit prostheses have been those who had all five digits amputated with remaining metacarpal/palm anatomy. Partial hand systems are difficult to incorporate for daily use and further research is needed to examine the factors involved.

\section{New Therapies and Research}

There are a number of advanced prostheses that are in development, thanks in part to Defense Advanced Research Projects Agency's (DARPA) research program "Revolutionizing Upper Extremity Prosthetics Program" launched in 2005 [15]. The intended goal is to improve upper extremity prostheses to allow greater function by utilizing the latest technological advancements. The study has promoted research involving complex collaborations for advancements such as incorporating sensation through the peripheral and central nervous system. Two of these terminal devices are the Modular Prosthetic Limb (MPL) and the DEKA arm. Time will determine what products become commercially available, are reimbursable, and provide the most benefit to those with upper extremity amputations.

New control schemes are being further researched for effectiveness. Implantable MyoElectric Sensors (IMES) have recently been surgically implanted into various muscles in the residual limb to achieve more accurate reception of myoelectric signal and may serve to add more intuitive control of the myoelectric prosthesis [16]. The electrodes communicate wirelessly through the skin when exposed to a coil embedded in the socket. Targeted Muscle Reinnervation (TMR) is a surgical procedure that was developed at the Neural Engineering Center for Artificial Limbs (NECAL) at the Rehab Institute of Chicago and has been performed on multiple transhumeral patients at WRNMMC [17]. This procedure splits and relocates the distal nerves that control the hand to other de-innervated muscle. The muscle acts as an amplifier for this signal from more intuitive nerves such as median, radial, and ulnar nerves. If this surgery results in an optimal outcome, multiple myoelectric sites will exist in the transhumeral residual limb. However, the individual outcomes may vary. Occasionally, distinct individual signals are not consistent, or some myosites may be stronger than others. One very promising effect of this procedure is that pain and neuromas can be greatly reduced [17]. This decrease in pain may be due to the reintegration of the previously severed nerves that are now essentially intact and not eliciting a pain response. Additionally with this procedure, a few patients have undergone an osteo-angulation, in which the humerus is fractured and angled with internal hardware, so as to allow for improved socket fit and suspension. These are promising advancements that need further research and development that could be new standards of practice.

There are new developments in commercially available myoelectric controls. CoApt is a pattern recognition socket system and software program that serves the transhumeral and transradial amputation levels [18]. Instead of use of traditional sensors placed over specific muscle bellies to perform isolated contractions, multiple sensors are placed throughout the prosthetic socket. This allows for intuitive control of the prosthesis in which the user naturally attempts the movements of the phantom arm and the electrodes measure the strength and pattern of the muscle contractions used. This can be used in conjunction with TMR. The prosthesis is controlled based off of the pattern of the muscle contractions that were read. The CoApt Pattern Recognition system stores these patterns of muscle contractions. The prosthesis is programed through a series of movements when the user first puts the socket on and can be reprogramed when the user feels like control is waning. It was developed in cooperation with Dr. Todd Kuiken and NECAL laboratory at the Rehab Institute of Chicago and has demonstrated significant initial results.

The MPL utilizes the advanced robotics and software collaboration of Applied Physics Lab and Johns Hopkins University for pattern recognition [19]. This device offers 26 degrees of freedom, 17 motors, and many additional sensors to provide greater function to the user. The MPL device is an advanced robotic terminal device that uses multiple electrodes through pattern recognition to offer versatile performance. Consistent control and overall weight of the prosthesis have been the primary limitations with this device, per patient report. This prosthesis is currently in the testing phases and is not yet commercially available. This MPL arm is promising technology that needs further research and development to create a viable device.

The DEKA arm [20] has been in research with the VA health care system for 6 years. The DEKA arm has ten powered degrees of freedom, which allow simultaneous control and offer unique control systems. This terminal device is controlled with the use of Inertial Measurement Unit (IMU's) which are attached to the wearer's shoe, and sense where the foot is in space. Position and motion of the foot operates the terminal device, as well as a switch worn on the body [20]. This device has been approved for marketing by the Food and Drug Administration as of May 2014 [15]. The DEKA arm offers many new control features and is a potential opportunity for amputees in the future.

The most complex medical development related to upper extremity amputations has been the ability of medical teams to successfully perform arm transplants [21]. Recently, the US military has supported the initiative to provide bilateral arm transplants to retired SGT Brendan Marrocco [22]. SGT Marrocco is the first quadruple 
amputee injured in the Iraq/Afghanistan conflicts in April 2009. After extensive medical testing, John Hopkins University Hospital in Baltimore, Maryland approved SGT Marrocco to be on the transplant list until a donor was available. In December 2012, SGT Marrocco received a bilateral upper extremity transplant by Dr. Andrew Lee and an extensive surgical team. SGT Marrocco continues to make progress in daily therapy with increasing motor control and decreasing pain. Arm transplants require intensive OT rehabilitation, for a minimum to $4 \mathrm{~h}$ per day for 2 years. It is expected that gains will continue beyond that time frame. As this is a complex process, upper extremity transplants require a significant investment in time, resources, and patience. When more transplants are performed, knowledge will increase and medical teams will be able to more accurately predict outcomes, and further develop this procedure as a viable option for amputees.

There are some more traditional prosthetic options to improve suspension as well. Osseointegration offers improved suspension options but potentially increases the risk of infection [23]. To date, this suspension method has been primarily performed in Europe and awaits further research in the US. Angulation osteotomy of the humerus offers improved suspension for a transhumeral amputation in which suspension is potentially possible without chest harnessing and allows for internal and external rotation of the shoulder [24]. A humeral $\mathrm{T}$ prosthetic bone implant would also provide a condylar-type suspension, but needs further research as well [25]. The above procedures could improve suspension and performance, but need further research to become standards of practice.

\section{Prosthetic Improvements Needed}

Upper extremity prostheses are designed to replicate and replace hand function, however, the complexity and versatility of hand anatomy make prosthetic design especially challenging. Despite recent advancements in technology, the main issues continue to be method of control and feedback from the prosthesis. To truly replicate the human hand, prostheses need to have a method of control that allows improved accuracy, dexterity, and provides sensory feedback to the user. Technological advances have not yet achieved these levels of human interface.

Myoelectric devices are susceptible to inadvertent movements, and there are few options for customization. BeBionic and Touch Bionics released software to allow the user to independently change controls of the prosthesis. Touch Bionics has incorporated phone technology to allow patients to select settings for grips and methods of control. However, improved methods to control the sensitivity of prostheses, such as adjusting firmness of grip, would allow the patient have increased security in their prosthetic control. Additionally, ease of access to sensitivity controls would allow the patient to quickly transition to settings that allow smoother performance of tasks. Overall, myoelectric devices could be further developed with technological advances such as new socket designs, improved cosmetics, intuitive control, increased battery life, water proofing, decreased weight, and ease of access for sensitivity controls, power switches, and charging.

There are also a variety of improvements that need to be made in terms of the versatility of terminal devices, in order to improve performance and decrease the need for frequent switching of devices. Multiple limb amputees who use wheelchairs would benefit from a terminal device that offers a functional pinch and still provides a consistent propulsion surface. Improvements made for hook terminal devices such as coverings that could disperse the pressure from tines would allow the ability to hold soft items without crushing them. The availability of three dimensional printers could benefit this population as patients are able to design terminal devices to meet their specific needs. A Chicago-based company e-Nabler has fabricated a variety of these printed devices for public use [26]. Quick release options to change individual tines or fingers would also be useful. No terminal device can accomplish all daily tasks encountered, but a design optimized for addressing individualized needs would be beneficial.

One major needed area of development would be accessing technology such as cell phones. Cell phones create challenges for prosthesis users, especially as cell phones continue to be an essential part of daily life. The main demand for prosthetic advancement would be to allow users to access touch screens. A stylus incorporated into a prosthetic terminal device or a mild electrical charge at a prosthetic fingertip would allow access to touch screen phones, ATMs, or computers. Currently, touch screens pose a daunting challenge for bilateral upper extremity amputees, especially without a stylus with an internal power source. Terminal devices need to become more technologically friendly in order for those with upper extremity amputations to interact successfully in the community.

Additional improvements could be made for cosmetic sleeves and gloves that are fabricated to cover the mechanical areas of prostheses. As terminal devices need to be rigid to prevent items from slipping, sometimes cosmetic covers can make tasks difficult. Technologies such as anti-motion sensors which increase pinch force are often more of a hindrance than an assist as they squeeze objects out of the hand. Terminal devices that are more cosmetically appealing could eliminate the need for cosmetic gloves and allow for improved accuracy. 


\section{Research Needed}

Research is limited in the rehabilitation and management of patients with upper extremity amputations. Research questions drive practice in rehabilitation by assisting in the facilitation of improved technological advancements in prosthetics and the training protocols that accompany them. Areas for research and improvement are found in all phases of upper extremity prosthesis rehabilitation.

In the acute phase, effective strategies for managing both residual limb pain and phantom limb pain, continues to be an area of research. Pain is an individualized experience that limits progress in prosthetic fit and training. An area of exploration in pain research would be how the concept of phantom limb sensation and the ability of the patient to "move" this phantom limb could be used to enhance prosthetic control, assist in recovery, and improve overall functional outcomes.

Upper extremity prosthesis acceptance rates vary and need further research to determine the factors involved [27]. Wright in a sample of 135 upper extremity amputations found an overall acceptance rate of $62 \%$, but this includes an acceptance rate of $94 \%$ for below elbow amputees. The acceptance rate for patients with transhumeral amputations was $45 \%$. Factors for rejection included limited usefulness, weight, and socket discomfort [27]. It is difficult to determine if different treatment, therapy, or prostheses may impact these acceptance rates. Would initial use of an immediate post-operative prosthesis (IPOP) device affect these numbers [28]? Would these rates change if the patient received the prosthesis within the golden period of 30 days as indicated by Malone [4]? There are many factors to consider when assessing prosthesis acceptance and further research is needed to determine how to improve outcomes.

In terms of the outpatient prosthetic training phase, there are currently few standardized tests available for assessing upper extremity prosthesis performance. The existing standardized OT assessments only assess hand performance and are not all normalized for prosthetic use and performance. Continued research in standardized testing and assessment for prosthetic use and performance is needed to gather objective functional outcome measures. In addition, there are numerous factors that OTs and prosthetists consider when providing prosthetic recommendations. Additional research and objective data to support those recommendations are necessary for practice, specifically research on the relationship that amputation level has on prosthetic preference. Determining the contributing factors to prosthesis use versus rejection will assist to guide prosthetic recommendations. Limited research has been performed to determine how upper extremity prostheses could affect gait. Does the type of upper extremity prosthesis, its suspension mechanism, and/or weight of the prosthesis have an effect on gait? Does the use of prostheses directly correlate with a one's energy expenditure, efficiency, and the presence of low back pain during gait? Evidence to support these questions would assist to drive therapy protocols and recommendations in training.

\section{Conclusion}

The conflicts in Iraq and Afghanistan have resulted in increased awareness and research in amputee rehabilitation. WRNNMC has worked to create a consistent and comprehensive amputee training program, resulting in several successful aspects of care that need formal research to determine outcomes. Although there are several technological advances in prostheses/control schemes and medical procedures, there is a significant need for OT assessments to measure functional prosthetic use and longterm outcomes. Additionally, the majority of the prosthetic issues remain the same; prosthetic acceptance rates are generally low [27] for upper extremity patients, the initial 30 days are a large predictor of prosthetic success [4], and prosthetic selection is incredibly individualized. Determining what prostheses are appropriate to meet the patient's need still remains challenging. The variability of acceptance indicates that there is a need for improved treatment and/or improved prosthetic options. Research needs to continue across a variety of variables in order to improve prosthetic design, treatment, and outcomes for the amputee population.

\section{Compliance with Ethics Guidelines}

Conflict of Interest J Butkus, C Dennison, A Orr and M St Laurent all declare no conflicts of interest.

Human and Animal Rights and Informed Consent This article does not contain any studies with human or animal subjects performed by any of the authors.

\section{References}

Recent publications of particular interest have been highlighted as:

• Of major importance

1. Military Amputee Patient Care Program Database. Extremity Trauma and Amputation Center of Excellence. Retrieved from: http://informatics-prod.mamc.amedd.army.mil/EACE/. Accessed on 1 July 2014.

2. - Smurr LM, Gulik K, Yancosek K, Ganz O. Managing the Upper Extremity Amputee: a Protocol for Success. J Hand Ther. 2008;21(2):160-76. This reference outlines and details the 
phases of upper extremity amputee care and how occupational therapy intervenes and benefits the patient population.

3. Chan BL, Witt R, Darrow AP, Howard R, Pasquina PF, Heilman KM, et al. Mirror therapy for phantom limb pain. N Engl J Med. 2007;357:2206-7.

4. Malone JM, Fleming LL, Roberson J. Immediate, early, and late postsurgical management of upper-limb amputation. J Rehabil Res Dev. 1984;21(1):33-41.

5. - Atkins JD, Meier RH, editors. Comprehensive Management of the Upper-Limb Amputee. Springer-Verlag: New York; 198948. This reference details how occupational therapy plays a role in the care of the upper limb amputee.

6. Wright V. Prosthetic outcome measures for use with upper limb amputees: a systematic review of the peer-reviewed literature, 1970 to 2009. J Prosthet Orthot. 2009;21(4):3-63.

7. Hermansson L, Fisher A, Bernspang B, Eliasson AC. Assessment of capacity for myoelectric control: a new rasch-built measure of prosthetic hand control. J Rehabil Med. 2005;37(3):166-71.

8. Jebsen RH, Taylor N, Trieschmann RB, Trotter MJ, Howard LA. An objective and standardized test of hand function. Arch Phys Med Rehabil. 1969;50(6):311-9.

9. Mathiowetz V, Volland G, Kashman N, Weber K. Adult norms for the Box and Block Test of manual dexterity. Am J Occup Ther. 1985;39:386-91.

10. Mathiowetz V, Weber, Kashman N, Volland G. Adult norms for the Nine Hole Peg Test of finger dexterity. Occup Ther J Res. 1985;5:24-33.

11. Light CM, Chappell PH, Kyberd PJ. Establishing a standardized clinical assessment tool of pathologic and prosthetic hand function: normative data, reliability, and validity. Arch Phys Med Rehabil. 2002;83:776-83.

12. Vincent hand. 2014. Retrieved June 30, 2014 from http://www. handprothese.de/evolution $2 /$.

13. Vincent finger. 2014. Retrieved June 30, 2014 from http://www. handprothese.de/vincent-finger/.

14. M-fingers. 2014. Retrieved June 30, 2014 from http://www. liberatingtech.com/products/hands/M-Fingers___Partial_M-Fingers_ for_Partial_Hands.asp.

15. Defense Sciences Office. 2014. DARPA: Revolutionizing prosthetics program. Retreived June 30, 2014 from http://www.darpa. mil/Our_Work/BTO/Programs/Revolutionizing_Prosthetics.aspx.

16. Weir RF, Troyk PR, DeMichele G, Lowery M, Kuiken T. Implantable Myoelectric Sensors (IMES). In: Proceedings from
MyoElectric Controls/Powered Prosthetics Symposium. Fredrickton: MEC'05 Integrating Prosthetics and Medicine; 2005.

17. Kuiken TA, Schultz Feuser AE, Barlow AK. Targeted Muscle Reinnervation: A Neural Interface for Artificial Limbs. Boca Raton: Taylor \& Francis Group; 2014.

18. CoApt. 2014. Retrieved June 30, 2014 from http://www. coaptengineering.com/pattern-recognition.html.

19. Johannes MS, Bigelow JD, Burck JM, Harshbarger SD, Kozlowski MV, Van Doren T. An overview of the developmental process for the modular prosthetic limb. Johns Hopkins APL Tech Digest. 2011;30(3):207-16.

20. Resnik L, Klinger SL, Etter K. User and clinical perspectives on DEKA Arm: results of VA study to optimize DEKA arm. J Rehabil Res Dev. 2014;51(1):27-38.

21. Schneeberger S, Landin L, Jableki J, Butler P, Hoehnke C, Brandacher G, ESOT CTA Working Group. Achievements and challenges in composite tissue allotransplantation. Transpl Int. 2011;24:760-9.

22. Ruane Michael. With Transplanted Arms and Army Grit, a Quadruple Amputee Soldiers On. The Washington Post. 2014. Retrieved June 30, 2014 from http://www.washingtonpost.com/local/withtransplanted-arms-and-army-grit-a-quadruple-amputee-soldiers-on/ 2014/06/30/5130c242-f6e8-11e3-a3a5-42be35962a52_story.html.

23. Jonsson S, Caine-Winterberger K, Branemark R. Osseointegration amputation prostheses on the upper limbs: methods, prosthetics and rehabilitation. Prosthet Orthot Int. 2011;35(2):190-200.

24. Marquardt E, Neff G. The angulation osteotomy of above-elbow stumps. Clin Orthop Relat Res. 1974;104(23):232-8.

25. Wits $\emptyset$ E, Kristensen T, Benum P, Sivertsen S, Persen L, Funderud A, Magne T, Aursand HP, Aamodt A. Improved Comfort and Function of Arm Prosthesis After Implantation of a Humerus-TProsthesis in Trans-Humeral Amputees. Prosthet Orthot Int. 2006;30(3):270-8.

26. E-nabler 2014. Retrieved June 30, 2014 from http:// enablingthefuture.org/.

27. Wright TW, Hagen AD, Wood MB. Prosthetic Usage in Major Upper Extremity Amputations. J Hand Surg. 1995;20(4):619-22.

28. Malone J, Childers S, Underwood J, Leal J. Immediate postsurgical management of upper extremity amputation: conventional, electric and myoelectric prosthesis. Orthot Prosthetics. 1981; 35(2):1-9. 